\title{
Evaluación del diagnóstico clínico, la aspiración citología con aguja fina y la mamografía, en lesiones palpables de la glándula mamaria
}

\author{
María Clemencia Ceballos, Constanza Díaz, Eduardo Serna, Jaime Uribe, Alejandro Sannin*
}

\begin{abstract}
RESUMEN: EI análisis de 322 lesiones palpables de la mama, con estudio histo-patológico, mostró alta sensibilidad, especificidad, valor predictivo positivo y valor predictivo negativo, a la combinación del diagnóstico clínico y la aspiración citología con aguja fina, y a la de éstas con la mamografía.
\end{abstract}

PALABRAS CLA VES: Carcinoma, y lesiones benignas palpables de la mama, citología por aspiración con aguja fina, diagnóstico clínico, mamografía.

SUMMARY: The analisis of 322 palpable lesions of mama with histopatological studies, showed high sensibility, specifity, predictive positive and negative value with the combination of clinical diagnostic and bacaf, and this combination with mammografy.

KEY WORDS: Carcinoma, bening palpable lesions, bacaf, clinical, diagnosis, mammografy.

\section{Introducción}

Tradicionalmente en nuestro medio la biopsia continua siendo la forma casi excluyente de diagnosticar el cáncer mamario palpable, lo cual genera morbilidad y ansiedad para los pacientes, altos costos para las instituciones, ausentismo laboral y una rata baja de afectividad.

Está reportado un gran número de estudios europeos y norteamericanos sobre la efectividad del diagnóstico citológico de la glándula mamaria, tanto de la patología benigna como de la maligna, alcanzando para muchos la categoría de un estudio histo-patológico (1-3). Los centros de referencia europeos informan una sensibilidad del 90 al 98\%, una especificidad del 98 al $100 \%$ y un UPP del más del $98 \%$ además de tener normatizada su utilización (4).

Otros estudios importantes evalúan los resultados de asociar el diagnóstico clínico y la citología con la mamografía en la llamada triple prueba diagnóstica de las lesiones palpables de la glándula mamaria con resultados casi del 100\% de efectividad (5-6).

En nuestro medio, la mamografía irrumpió sólo en esta última década, y la citología por aspiración con aguja fina a nivel institucional e involucrada en un protocolo, hace tres años, en la consulta de patología mamaria de la institución X. Estos dos elementos se han entrado a complementar el diagnóstico del clínico, quien determina en últimas cuáles lesiones deben someterse a biopsia y cuáles a seguimiento, con la gran responsabilidad que le corresponde para no retardar el diagnóstico de un carcinoma.

Un estudio retrospectivo de una experiencia inicial en el diagnóstico de lesiones palpables de la mama se emprendió para conocer la efectividad del diagnóstico clínico, de la citología por aspiración con aguja fina y de la mamografía con base en el resultado de histo-patología.

Ginecólogos y citopatólogos de una consulta institucional de patología mamaria. U. Antioquia

\section{Materiales y métodos}

Se presenta la experiencia del diagnóstico de masas palpables de la mama en una consulta institucional de patología mamaria.

\section{Aspiración citología con aguja fina}

1.117 aspiraciones para citología con aguja fina ACAF de lesiones palpables de las glándulas mamarias en 1.090 mujeres se realizaron en forma consecutiva, entre enero 2 de 1993 y septiembre 30 de 1995. El total de consultantes fue de 3.238 , y se puncionaron nódulos, o placas, o ambas, correspondiendo los primeros a la lesión tridimensional y al seudonódulo y las segundas a engrosamientos y asimetrías de interés clínico.

La aspiración se practicó en la primera visita de la paciente al consultorio. Se le explicó el método y se tomó su consentimiento. Las lesiones se aspiraron con una jeringa de 5 cc ensamblada a una aguja de una pulgada, calibre No. 24. Algunas veces, para lesiones profundas utilizamos aguja No. 22 de $11 / 2$ pulgada. No se utilizó anestesia local. Dos o tres pasos por la piel fueron seguidos de 10 o más pasos de la aguja a través de la lesión. El material aspirado fue inmediatamente expelido sobre una lámina portaobjetos nueva y esparcido con otra lámina en una capa homogénea y delgada hasta agotar el material -generalmente en dos o tres láminas-, que fueron colocadas también inmediatamente en inmersión en Ethanol al $95 \%$ durante media hora para fijar el material. Se utilizó la coloración de Papanicolau en diversas modificaciones.

En una primera fase del estudio, comprendida entre enero 2 de 1993 y agosto 31 de 1994, 337 ACAF fueron leídas por el patólogo Doctor Juan Manuel González en el laboratorio de Patología de la Clínica León XIII del ISS 84 por el patólogo Doctor B en su laboratorio particular. A partir de esta fecha, se crea el servicio en la institución $\mathrm{X}$, y la misma citopatóloga lee e informa lo que en primera instancia evalúan las citotecnólogas. Un total de 610 citologías fueron leídas en la institución X y este período se denomina la segunda fase del estudio. 
Las categorías diagnósticas para la ACAF se informaron, en el primer período del estudio (enero 2 de 1993 a septiembre 1 de 1994), en varios términos no estandarizados, que no especificaban a qué entidad histopatológica benigna podían corresponder realmente. Se procedió entonces a revisar los informes de las citologías y a reclasificarlas de la siguiente manera:

- Benigno: Manera de informar que se refiere solamente a la ausencia de cáncer pero no especifica el tipo de enfermedad benigna, si la hay.

- Enfermedad proliferativa benigna: Incluye las "compatibles con mastopatía fibroquística", las que en el informe especifican algún tipo de proliferación epitelial incluyendo la metaplasia apococrina, las hiperplasias, los quistes y la "papilomatosis" que hace referencia también a una hiperplasia.

Para esta reclasificación se tuvieron en cuenta los criterios del Doctor Haagensen (7) que agrupa dentro de la enfermedad proliferativa benigna a los quistes, la metaplasia apocrina, las adenosis y las hiperplasias. Igualmente se tuvieron en cuenta los criterios de Page (8) para clasificación e interpretación pronóstica de las hiperplasias e hiperplasias atípicas y a Rosen (9) quien cita a los dos autores anteriores.

- Atipias epiteliales: Las hiperplasias atípicas y las atipias "leves" que no hacen referencia a fenómenos de hiperplasia. En esta categoría se incluyen también los "fibroadenomas con atipias".

- Fibroadenoma: El fibroadenoma con frecuencia asociado a metaplasia apocrina o hiperplasia, se clasifica con el fibroadenoma simple.

- Inflamatorio por la presencia de polimorfonucleares, histiocitos, necrosis, células gigantes o linfocitos, espectro de la reacción inflamatoria.

- Maligno y sospechoso: No hubo ninguna reclasificación.

- Insuficiente: Comprende las muestras sin células epiteliales del conducto o del lobulillo mamario. Con frecuencia informaron grasa, fibroplastos o sangre.

- Mama: Elementos estromales y epiteliales que no resultan suficientes para hacer posible un diagnóstico de la entidad, pero que nos dan la certeza de que se entró al tejido mamario. Algunas veces los hallazgos se informaron como "mama normal".

\section{El diagnóstico clínico}

Se tomaron en cuenta los diagnósticos definitivos consignados en las historias, pero no se definió una variable para el análisis llamada Diagnóstico clínico final, consistente en: El resultado de anatomía patológica si lo tuvo; el resultado de la ACAF si fue diferente o si precisaba más el diagnóstico inicial, por ejemplo un cambio fibroquístico pasó a ser macroquiste o fibrosis.

En los casos en los cuales no existieron estas dos situaciones, se conservó el diagnóstico clínico inicial.

\section{La mamografía}

Una mamografía se ordena en nuestro servicio, a algunas mujeres entre 35 y 40 años, a todas las mayores de 40 , y a las lesiones clínicamente sospechosas, o con citología maligna o sospechosa, no importando la edad. Para los nódulos pequeños y placas se recomienda que el estudio de mamografía se lleve a cabo dos semanas después de la punción aspiración para evitar imágenes anormales debidas al trauma del tejido.

Las mamografías fueron reportadas por distintos servicios de la ciudad en especial por dos radiólogos de las Clínicas W y Z (Doctores C y D).

Las categorías diagnósticas de las mamografías son: I mamografía normal, II lesión benigna, III lesión indeterminada probablemente benigna, IV probablemente maligna (sospechosa), $V$ sugestiva de malignidad. La categoría benigna corresponde a aquellos casos en que no se especificó en la historia clínica si se trataba de las categorías I, II ó III.

\section{La encuesta}

Una encuesta telefónica hecha por la Trabajadora Social de la institución X, y por uno de los investigadores, se aplica actualmente a dos grupos de pacientes: A aquellas cuya ACAF fue maligna y no se biopsió en la institución X. Se interrogó sobre el tipo de tratamiento recibido (mastectomía, radioterapia, quimioterapia u hormonoterapia), sobre el tipo de biopsia hecha antes del tratamiento -si la tuvo-y se trató de obtener copias de los resultados de histo-patología de las biopsias, mastectomías o cuadrantectomías. Se consignó además si falleció.

Un segundo grupo encuestado corresponde al de las pacientes con fibroadenomas o cambios fibroquísticos, CFQ u otras lesiones benignas que tuvieron ACAF, no dadas de alta. Se encuestaron con el siguiente protocolo: los entrevistadores preguntan a la paciente acerca de la lesión mamaria aspirada, especialmente si la sabe localizar por autoexamen y si nota algún cambio. Si la sabe localizar y no nota ningún cambio en la lesión o ésta "desapareció", se considera estable. Si alguno de los criterios no se cumple se cita a seguimiento clínico.

De esta manera se están recuperando las pacientes que no asistieron a los controles, o se están dando de alta por encontrarse perdidas.

\section{El seguimiento clínico}

Consiste en reevaluar clínicamente la lesión, medirla en milímetros con un instrumento de medición precisa, determinar y consignar en la historia si aumentó, disminuyó, desapareció o parece estable con respecto a la medición anterior. Repetir la ACAF en los casos en que el reporte de la primera fue una enfermedad proliferativa, o cuando la lesión creció, o cuando la primera aspiración resultó insuficiente y la lesión persiste. Se consigna la sintomatología relacionada con la lesión y se reevalúan el diagnóstico y la conducta.

La mamografía de control no está protocolizada y queda a criterio clínico.

Una resección quirúrgica está indicada por sospecha de CA, crecimiento importante de la lesión, síntomas asociados o deseo de la paciente. 
Los resultados del seguimiento presentados en este trabajo, corresponden a los afectados hasta diciembre 20 de 1995.

\section{La historia clínica}

Para este estudio se utilizó una historia clínica precodificada, en la que se analizó el tipo de lesión "nódulo" o "placa densa", el diagnóstico clínico, los hallazgos mamográficos, el resultado de la ACAF, el diagnóstico histológico y los datos del seguimiento clínico.

Para el análisis de los tres métodos diagnósticos, diagnóstico clínico, ACAF y mamografía se calcularon los siguiente valores relativos: Sensibilidad, especificidad, valor predictivo positivo (VPP) y valor predictivo negativo (VPN) correlacionando las categorías diagnósticas de cada prueba, con los diagnósticos histopatológicos. Tabla 1.

Se consideraron negativos para malignidad, los informes de ACAF benigno, enfermedad proliferativo benigna, atipias epiteliales, fibroadenoma e inflamatorio; los informes mamográficos benigno, categorías I, II, III, los informes histo-patológicos diferentes a cáncer y tumor Phyllodes maligno; y los diagnósticos diferentes a CA de mama, sospecha de CA, tumor Phyllodes y enfermedad de Paget, los cuales se consideraron positivos para malignidad, junto con los diagnósticos citológicos maligno y sospechoso, y las mamografías categorías IV y V.

\section{Análisis y resultados}

Las 1.116 lesiones palpables de 1.089 mujeres produjeron 1.031 informes de citología aspirativa (85 punciones no fueron informadas). Un diagnóstico citológico no fue posible en el $35.8 \%$ de los $\mathrm{ACAF}$, porque fueron informados como "mama" (17\%) e insuficiente (18.8\%).

De estas pacientes, en 322 se tuvo conocimiento sobre diagnóstico histo-patológico proveniente de: 279 biopsias hechas en la institución X, tres biopsias hechas en otros

\section{Tabla 1}

ANALISIS DE VALORES RELATIVOS

\begin{tabular}{|ll|}
\hline Valor relativo & \multicolumn{1}{c|}{ Definición (No. casos) } \\
\hline Sensibilidad & $\begin{array}{l}\text { Verdaderos positivos } \\
\text { Verdaderos positivos + falsos } \\
\text { negativos }\end{array}$ \\
Especificidad & $\begin{array}{l}\text { Verdaderos negativos } \\
\text { Verdaderos negativos + falsos } \\
\text { positivos }\end{array}$ \\
Valor predictivo & $\begin{array}{l}\text { Verdaderos positivos } \\
\text { Perdaderos positivos + falsos } \\
\text { positivos }\end{array}$ \\
Valor predictivo & $\begin{array}{l}\text { Verdaderos negativos } \\
\text { Verdaderos negativos + falsos } \\
\text { Negativo }\end{array}$ \\
& negativos \\
\hline
\end{tabular}

hospitales, y de 40 pacientes con ACAF diagnóstica de malignidad remitidas para tratamiento definitivo a otras instituciones y en quienes éste se conoció por medio de la encuesta telefónica. El informe escrito de la histopatología de la mastectomía o cuadrantectomía no fue posible lograrlo en la totalidad de los casos.

602 mamografías fueron informadas de las 1.116 lesiones. De un total de 459 mujeres mayores de 40 años con lesión palpable el $18.7 \%$ de ellas no se practicó el examen indicado.

De 322 pacientes con manejo quirúrgico a 213 se les practicó mamografías. Las indicaciones para realizar biopsia según el resultado de la mamografía dependió en parte la interpretación que el clínico hizo de las imágenes según los hallazgos al examen físico y a la ACAF, por lo que no todas las mamografías sospechosas fueron biopsiadas. Tabla 2 .

Un comentario especial merece el alto porcentaje de citologías no diagnósticas. La literatura ha reportado márgenes amplios para las muestras insuficientes, de 6 a $32 \%$ (10) y ha mencionado para los centros de referencia de patología maligna un máximo permisible de un 5,0\% para asegurar un buen control de calidad. Sin embargo, abundantes referencias han aparecido recientemente sobre el papel de la ACAF en la valoración de la patología benigna. Una serie importante reportó un $6.5 \%$ de muestras insuficientes para la patología tumoral maligna y un $23.2 \%$ para la patología benigna (11).

En este estudio se deben considerar, primero, que se aplicó ampliamente la ACAF, a la valoración de toda la patología benigna y en el caso del C.F.Q., no solamente al nódulo dominante sino también a placas densas asimétricas y a engrosamientos más discretos; y segundo, que hubo errores en la aplicación de la técnica de aspirado, en la coloración y en la lectura de las placas, generadas por un equipo de trabajo que en la primera fase del estudio no tenía experiencia en la ACAF de la glándula mamaria.

La tabla 3 nos muestra los diagnósticos histológicos que nos darán el respaldo para evaluar nuestros tres métodos diagnósticos: Diagnóstico clínico, ACAF y mamografía.

El promedio de edad de las pacientes en cada uno de los diagnósticos finales se muestra en la Tabla 4.

Para el carcinoma, la edad promedio fue de 51.7 años, lo que concuerda con lo descrito por Haagensen para cánceres palpables (12). De 129 carcinomas refrendados por histo-patología, en las 1.116 lesiones, sólo hubo cuatro carcinomas intraductales (el $3.1 \%$ ), frecuencia igual a la presentada en la misma referencia que estudió una serie norteamericana de los años 1931 a 1972. Las edades promedio para el grupo de los FA, los quistes y la enfermedad fibrosa, también coinciden con el autor. En un reporte más reciente se encontraron los mismos promedios de edad para el CA y FA que los muestreos y se informa además una media de la edad para la CFQ que coincide con la presente serie (13).

Agrupamos la ectasia ductal y la galactoforitis con o sin absceso para poder confrontar las lesiones inflamatorias encontradas con los resultados de las ACAF.

La información de los diagnósticos clínicos frente a los informes de piezas quirúrgicas se presenta en la Tabla 5, 
Tabla 2

FRECUENCIA DE LAS CATEGORIAS DIAGNOSTICAS EN LOS TRES METODOS ESTUDIADOS

\begin{tabular}{|c|c|c|c|c|c|c|c|c|}
\hline $\begin{array}{l}\text { Diagnóstico clínico } \\
\text { Inicial }\end{array}$ & $\begin{array}{l}\text { No. } \\
\text { Casos }\end{array}$ & $\%$ & $\begin{array}{l}\text { Citología } \\
\text { Aspiración con } \\
\text { aguja fina }\end{array}$ & No. & $\begin{array}{c}\% \\
\text { Casos }\end{array}$ & Mamografía & $\begin{array}{l}\text { No. } \\
\text { Casos }\end{array}$ & $\%$ \\
\hline CA de mama & 181 & 16.2 & Atipias & 29 & 2.8 & Benigna & 149 & 24.8 \\
\hline FA & 352 & 31.5 & Maligno & 115 & 11.2 & I & 73 & 12.1 \\
\hline CFQ & 406 & 36.4 & Sospechoso & 5 & 0.5 & II & 123 & 20.4 \\
\hline Macroquiste & 45 & 4.0 & EPB & 176 & 17.1 & III & 35 & 5.8 \\
\hline Ectasia-mastitis & 29 & 2.6 & ENP & 38 & 3.7 & IV & 89 & 14.8 \\
\hline Papiloma & 9 & 0.8 & Fibroadenomas & 156 & . & V & 62 & 10.3 \\
\hline Enfermedad fibrosa & 4 & 0.4 & Inflamatorio & 34 & 3.3 & Total & 602 & \\
\hline Lipoma & 10 & 0.9 & Benigno (soe) & 95 & 9.2 & $\begin{array}{l}\text { Mamografías en } \\
\text { pacientes con }\end{array}$ & 213 & \\
\hline T. Phyllodes & 8 & 0.7 & Papiloma & 11 & 1.1 & biopsias & & \\
\hline Sana & 27 & 2.4 & TPB & 3 & 0.3 & & & \\
\hline \multirow[t]{3}{*}{ En estudio } & 45 & 4.0 & TPM & 0 & 0 & & & \\
\hline & & & Mama & 175 & 17.0 & & & \\
\hline & & & Insuficiente & 194 & 18.8 & & & \\
\hline Total & 1116 & & Total & 1031 & & & & \\
\hline
\end{tabular}

$\mathrm{FA}=$ Fibroadenoma

$\mathrm{TPB}=\mathrm{T}$. Phyllodes benigno

$\mathrm{CFQ}=$ Cambio fibroquístico
TPM= T. Phyllodes malign

$\mathrm{ENP}=$ Enfermedad no prolif.
Tabla 3

FRECUENCIA DE LOS DIAGNOSTICOS DE HISTOPATOLOGIA

\begin{tabular}{|lcc|}
\hline Diagnóstico histopatológico & $\begin{array}{c}\text { No. de } \\
\text { casos }\end{array}$ & \% \\
\hline CA invasor & 117 & 36.3 \\
CA Intraductal & 4 & 1.2 \\
Enfermedad proliferativa benigna & 28 & 8.6 \\
Mastopatía fibroquística & 40 & 12.4 \\
Hiperplasia atípica & 5 & 1.5 \\
Fibroadenoma & 94 & 29.1 \\
Enfermedad fibrosa & 3 & 0.93 \\
Benigno & 8 & 2.4 \\
Inflamación & 9 & 2.7 \\
Lipoma & 2 & 0.6 \\
Papiloma & 7 & 2.1 \\
T. Phyllodes maligno & 5 & 1.5 \\
\hline Total & 322 & \\
\hline
\end{tabular}

Tabla 4

PROMEDIO DE EDAD EN RELACION CON EL DIAGNOSTICO FINAL

\begin{tabular}{|lc|c|c|c|}
\hline & & \multicolumn{3}{|c|}{ Edad en años } \\
\hline \multicolumn{1}{|c|}{$\begin{array}{c}\text { Diagnóstico } \\
\text { clínico final }\end{array}$} & $\begin{array}{c}\text { No. de } \\
\text { Casos }\end{array}$ & Rango & Media & D.S \\
\hline CA de mama & 162 & $29-85$ & 51,7 & 13,13 \\
Cambios fibroquísticos & 391 & $16-71$ & 36,5 & 9,14 \\
Fibroadenoma & 342 & $14-71$ & 32,1 & 9,30 \\
Mastitis-Galactoforitis- & & & & \\
Ectasia & 40 & $24-65$ & 38,6 & 9,11 \\
Fibrosis mamaria & 18 & $17-60$ & 37,7 & 10,75 \\
Macroquiste & 43 & $29-65$ & 44,6 & 7,36 \\
Lipoma o fibroadeno- & & & & \\
lipoma & 17 & $19-63$ & 42,4 & 13,62 \\
Papiloma & 10 & $18-66$ & 43,1 & 11,86 \\
Tumor Phyllodes & 9 & $21-61$ & 40,2 & 13,03 \\
Sanas & 62 & $11-65$ & 35,8 & 12,17 \\
Diferido & 22 & $24-67$ & 44,6 & 13,73 \\
\hline Total & 1116 & & \multicolumn{3}{|l}{} \\
\hline
\end{tabular}


Tabla 5

LESIONES PALPABLES MANEJADAS QUIRURGICAMENTE CORRELACION DEL DIAGNOSTICO CLINICO CON LA HISTO-PATOLOGIA

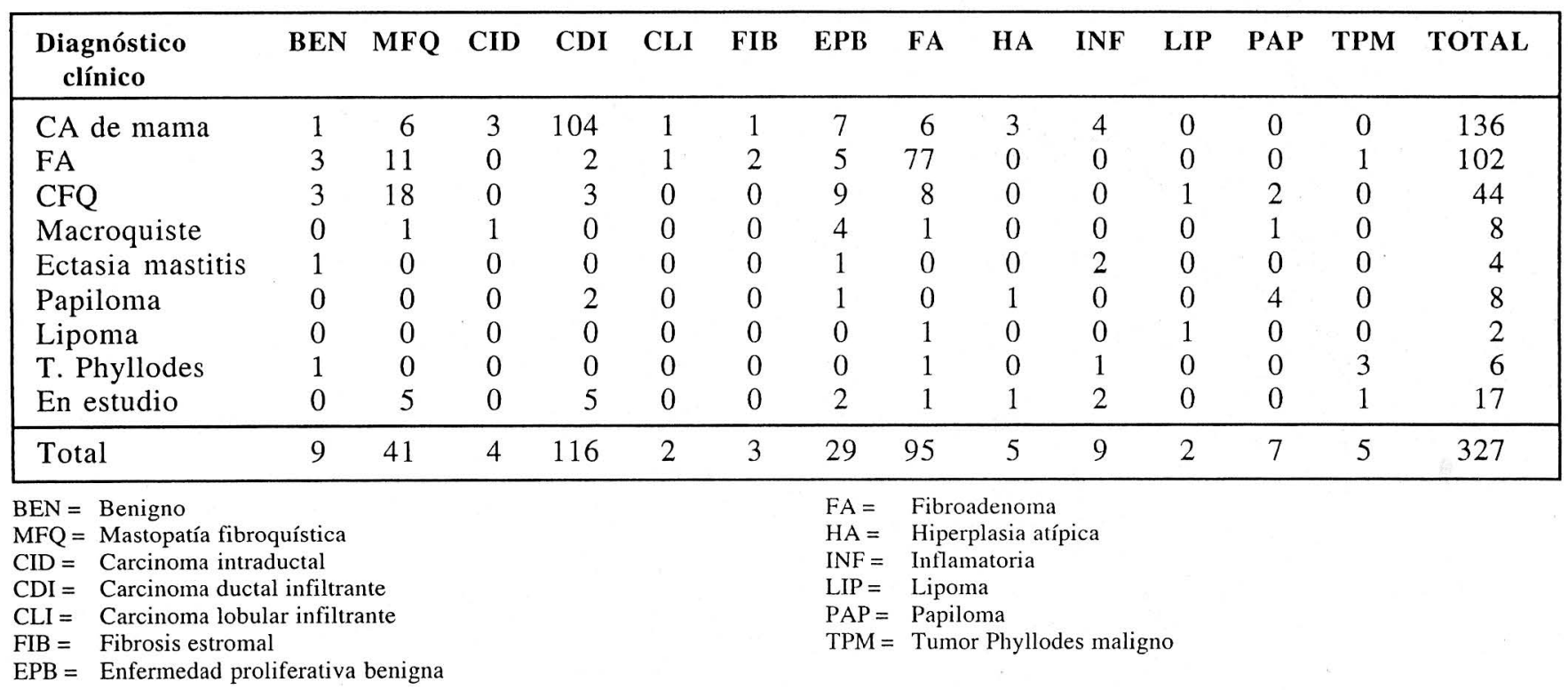

con 327 registros, y los resultados de la ACAF frente a los diagnósticos obtenidos de las piezas quirúrgicas, se presentan en la Tabla 6 con cinco registros menos, correspondientes a lesiones cuya ACAF no fue informada.

De entre los resultados no diagnósticos de las ACAF es importante resaltar que se obtuvieron 12 casos de carcinoma ductal infiltrante (igual proporción como mama o insuficiente), y 28 casos de fibroadenoma (24 reportados como mama y el resto como insuficiente).

La ACAF sirvió para diagnosticar correctamente el FA en el $38.2 \%$ de los casos reportados como tales en las piezas quirúrgicas y la biopsia fue concordante en el

Tabla 6

\section{LESIONES PALPABLES MANEJADAS QUIRURGICAMENTE CORRELACION CITOLOGIA/HISTO-PATOLOGIA}

\begin{tabular}{|c|c|c|c|c|c|c|c|c|c|c|c|c|c|c|c|}
\hline \multicolumn{2}{|c|}{$\begin{array}{l}\text { Diagnóstico } \\
\text { citológico } \\
\text { (por aspiración) }\end{array}$} & CDI & CLI & SIS & $\begin{array}{l}\text { CFQ } \\
\text { (soe) }\end{array}$ & EPB & HA & FA & FIB & INF & LIP & PAP & BEN & TPM & TOTAL \\
\hline \multicolumn{2}{|c|}{ Atipias } & 4 & 0 & 0 & 5 & 3 & 1 & 7 & 0 & 0 & 0 & 1 & 0 & 0 & 21 \\
\hline \multicolumn{2}{|c|}{ Maligno } & 89 & 2 & 2 & 1 & 0 & 0 & 0 & 0 & 0 & 0 & 0 & 1 & 1 & 96 \\
\hline \multicolumn{2}{|c|}{ Sospechoso } & 5 & 0 & 0 & 0 & 0 & 0 & 0 & 0 & 0 & 0 & 0 & 0 & 0 & 5 \\
\hline \multicolumn{2}{|l|}{$\mathrm{EPB}$} & 2 & 0 & 1 & 11 & 8 & 1 & 15 & 1 & 0 & 0 & 3 & 3 & 1 & 46 \\
\hline \multicolumn{2}{|c|}{ Fibroadenoma } & 2 & 0 & 0 & 4 & 1 & 1 & 36 & 0 & 0 & 0 & 0 & 0 & 1 & 45 \\
\hline \multicolumn{2}{|c|}{ Inflamatorio } & 0 & 0 & 0 & 1 & 0 & 0 & 2 & 0 & 5 & 0 & 0 & 1 & 0 & 9 \\
\hline \multicolumn{2}{|c|}{ Benigno (soe) } & 1 & 0 & 1 & 3 & 6 & 0 & 4 & 0 & 1 & 0 & 0 & 0 & 0 & 16 \\
\hline \multicolumn{2}{|c|}{ Papiloma } & 0 & 0 & 0 & 1 & 1 & 0 & 2 & 0 & 0 & 0 & 1 & 0 & 0 & 5 \\
\hline \multicolumn{2}{|l|}{ TPB } & 0 & 0 & 0 & 0 & 0 & 0 & 0 & 0 & 0 & 0 & 0 & 0 & 1 & 1 \\
\hline \multicolumn{2}{|l|}{ TPM } & 0 & 0 & 0 & 0 & 0 & 0 & 0 & 0 & 0 & 0 & 0 & 0 & 1 & 1 \\
\hline \multicolumn{2}{|l|}{ Total } & 103 & 2 & 4 & 26 & 19 & 3 & 66 & 1 & 6 & 0 & 5 & 5 & 5 & 245 \\
\hline \multicolumn{2}{|l|}{ Mama } & 6 & 0 & 0 & 6 & 5 & 1 & 24 & 1 & 1 & 1 & 2 & 3 & 0 & 50 \\
\hline \multicolumn{2}{|c|}{ Insuficiente } & 6 & 0 & 0 & 8 & 4 & 1 & 4 & 1 & 2 & 1 & 0 & 0 & 0 & 27 \\
\hline \multicolumn{2}{|l|}{ Total } & 12 & 0 & 0 & 14 & 9 & 2 & 28 & 2 & 3 & 2 & 2 & 3 & 0 & 77 \\
\hline \multirow{7}{*}{$\begin{array}{l}\mathrm{CDI}= \\
\mathrm{TPB}= \\
\mathrm{CLI}= \\
\mathrm{CID}= \\
\mathrm{CFQ} \text { (soe) }= \\
\mathrm{EPB}= \\
\mathrm{HA}=\end{array}$} & \multicolumn{6}{|c|}{ Carcinoma ductal infiltrante } & $\mathrm{FA}=$ & \multicolumn{4}{|c|}{ Fibroadenoma } & $\mathrm{TPB}=$ & \multicolumn{3}{|c|}{ Tumor phyllodes benigno } \\
\hline & \multicolumn{6}{|c|}{ Tumor Phyllodes benigno } & $\mathrm{FIB}=$ & \multicolumn{4}{|c|}{ Fibrosis estom } & $\mathrm{TPM}=$ & Tum & or phyll & les maligno \\
\hline & \multirow{2}{*}{\multicolumn{6}{|c|}{$\begin{array}{l}\text { Carcinoma lobular infiltrante } \\
\text { Carcinoma intraductal }\end{array}$}} & $\mathrm{TPM}=$ & \multicolumn{4}{|c|}{ Tumor Phyllodes maligno } & & & & \\
\hline & & & & & & & $\mathrm{INF}=$ & \multicolumn{4}{|c|}{ Inflamatorio } & & & & \\
\hline & \multicolumn{6}{|c|}{ Cambio fibroquístico (sin otra especificación) } & LIP $=$ & \multicolumn{4}{|c|}{ Lipoma } & & & & \\
\hline & \multicolumn{6}{|c|}{ Enfermedad proliferativa benigna } & $\mathrm{PAP}=$ & \multirow{2}{*}{\multicolumn{4}{|c|}{$\begin{array}{l}\text { Papiloma } \\
\text { Benigno }\end{array}$}} & & & & \\
\hline & \multicolumn{6}{|c|}{ Hiperplasia atípica } & $\mathrm{BEN}=$ & & & & & & & & \\
\hline
\end{tabular}


$75,0 \%$ de los casos con diagnóstico clínico de fibroadenoma. (Ver Tabla 5). El $19.6 \%$ de los FA diagnosticados por histopatología se informaron en la ACAF como un cambio proliferativo (hiperplasia o adenosis) y ocho diagnósticos citológicos de FA se acompañaron de hiperplasia. Dos de ellos resultaron ser CA: Un carcinoma lobular infiltrante y un carcinoma ductal infiltrante.

La literatura señala que el componente epitelial lobular del FA puede sufrir casi todos los cambios proliferativos como la adenosis, la metaplasia apocrina o los diversos grados de hiperplasia con o sin atipias, y por supuesto el carcinoma que en conclusión tiene una relación simplemente locativa con el FA. La aspiración con una aguja recoge más fácilmente componentes epiteliales que estromales en el FA y el diagnóstico citológico se hará con base en estos hallazgos, por lo que su presencia en mayor o menor cantidad puede determinar la efectividad del ACAF en el diagnóstico citológico del FA.

Los 21 casos de atipias consignados en la Tabla 6 corresponden al $72.4 \%$ de todas las atipias encontradas mediante ACAF. Entre las manejadas quirúrgicamente, el $33.3 \%$ correspondieron a FA, el $19,0 \%$ y el $4.7 \%$ a hiperplasia atípica.

De los 144 carcinomas diagnosticados por ACAF, 88 se biopsiaron en la institución X $(61.1 \%)$ y 56 se remitieron sin biopsia a otras instituciones para el tratamiento definitivo. De éstas, 40 contestaron la encuesta mencionada. La Tabla 7 incluye las 88 pacientes biopsiadas y las 40 encuestadas. Se excluyen para todo análisis, las 16 pacientes no encuestadas.

Se trata en la consulta de implementar la biopsia con aguja cortante tipo truc-cut, sin embargo se sabe que es una técnica que puede presentar complicaciones más serias que la ACAF. Algunos autores reportan la biopsia con truc-cut como menos efectivo que la ACAF para diagnosticar el cáncer (14). En este estudio, a 29 pacientes se les practicó ACAF con resultado maligno y posteriormente una biopsia con truc-cut; en tres de ellas (10.3\%), el truc-cut tuvo resultado falso negativo, mientras que en 19 de 126 cánceres diagnosticados por histopatología el resultado fue un falso negativo histo-patología, el resultado de la ACAF fue un falso negativo $(15,0 \%)$, discriminado en $10(7.9 \%)$ muestras insuficientes y 12 muestras compatibles con "mama".

Tres pacientes con ACAF reportaron complicaciones: Una mastitis aguda después de hacer la aspiración a un abultamiento periareolar que resultó ser una galactoforitis, un hematoma discreto que produjo equimosis, y una paciente que en un control posterior refirió haber sentido dolor intenso en el sitio de la punción.

Sólo una de 327 pacientes biopsiadas resultó con un carcinoma que no fue sospechado en el diagnóstico clínico inicial ni en la ACAF y tampoco por la mamografía:

Paciente de 44 años con diagnóstico clínico de macroquistes de la unión de los cuadrantes superiores de la mama derecha, aspirados en tres oportunidades. La ACAF de una placa residual a la aspiración de los macroquistes reportó hiperplasia epitelial. Nunca se palpó un nódulo sólido. La última aspiración de los macroquistes mostró un contenido sanguinolento, acelular en el análisis citológico. La mamografía fue informada con lesiones múltiples benignas compatibles con macroquistes. Se programó una biopsia que mostró un CA intraductal extenso variedad intramural. Y quedaron márgenes de resección comprometidos. Una mastectomía simple, confirmó el diagnóstico de $\mathrm{CA}$ intraductal.

En la tabla 7 se presentan las pacientes en quienes el diagnóstico clínico inicial se modificó luego de conocer el resultado de la patología (falsos positivos en relación con el diagnóstico inicial). La proporción presentada corresponde a cada uno de los diagnósticos clínicos iniciales. Es importante destacar los casos en los cuales el diagnóstico definitivo correspondió a cáncer: Tres de los 17 casos (17.6\%) en los cuales el diagnóstico clínico inicial fue CFQ y se modificó con la histo-patología resultando ser cáncer. La misma situación ocurrió con el diagnóstico clínico inicial de FA en tres de los 25 casos diagnosticados erróneamente (12.0\%) y en un caso (4.0\%) que resultó ser, finalmente, un tumor Phyllodes maligno. La mitad de los casos diagnosticados clínicamente en forma errónea como cáncer pasaron a ser, de acuerdo con la histo-patología, enfermedades no proliferativas o proliferativas benignas.

En tres cuartas partes de los casos con diagnóstico inicial de FA, el diagnóstico histológico se presentó como enfermedad proliferativa benigna o no proliferativa.

Todo esto nos corrobora el hecho informado en la literatura sobre el traslape importante que hay entre el diagnóstico clínico e histo-patológico de cáncer, FA y CFQ (15) y nos da idea de la importancia que la ACAF tiene para precisar el diagnóstico clínico, seleccionar las pacientes que se deben biopsiar, descubrir carcinomas no sospechosos clínicamente, y reconocer las pacientes que

Tabla 7

CORRELACION CLINICO HISTOLOGICA DE LOS DESACIERTOS EN EL DIAGNOSTICO CLINICO INICIAL DIAGNOSTICO HISTOLOGICO

\begin{tabular}{|lccccccccc|}
\hline $\begin{array}{l}\text { Falsos } \\
\text { Positivos }\end{array}$ & $\begin{array}{l}\text { No. de } \\
\text { Casos }\end{array}$ & $\begin{array}{c}\text { EPB } \\
\text { ENP }\end{array}$ & FA & CA & INF & LIP & PAP & TPM & BEN \\
\hline CA & 31 & $54.8 \%$ & $22.6 \%$ & - & $16.1 \%$ & 0 & 0 & - & $6.5 \%$ \\
CFQ & 17 & - & $47.1 \%$ & $17.6 \%$ & 0 & $5.9 \%$ & $11.8 \%$ & 0 & $\begin{array}{c}17.6 \% \\
12 \%\end{array}$ \\
FA & 25 & $72 \%$ & - & $12 \%$ & 0 & 0 & 0 & $4 \%$ & $12 \%$ \\
\hline
\end{tabular}


presentan hiperplasias severas o hiperplasias atípicas para aclarar el diagnóstico por histo-patología y posteriormente para ayudar en su seguimiento.

\section{Lesiones palpables con manejo quirúrgico}

Las indicaciones para un manejo quirúrgico o un manejo expectante de la patología benigna no se protocolizaron. Las pacientes fueron remitidas en gran parte para una biopsia y una ACAF que se les practicó en la primera consulta.

Al grupo de pacientes no remitido, se le practicó $\mathrm{ACAF}$ en la primera consulta y se decidió el manejo con base en varios aspectos: Para el caso de los FA, se programó una cirugía o se decidió controlar clínicamente de acuerdo con el dolor, al tamaño de la lesión y a la aceptación o no de seguimiento por parte de la paciente. Los cambios fibroquísticos sólo se biopsiaron por sintomatología persistente en una lesión francamente dominante, o para descartar CA. Las lesiones inflamatorias tipo absceso o fístula se trataron exhaustivamente antes de recurrir a la cirugía. Las lesiones con ACAF reportado como "atipias leves", con impresión clínica de benignidad y en mujeres jóvenes, se observaron. Las hiperplasias severas se biopsiaron. Las ACAF insuficientes se respiraron, si el dato se consideró importante y muy pocas pacientes rehusaron una segunda aspiración.

Pacientes con diagnóstico clínico, con ACAF y con mamografía compatibles con carcinoma, no se biopsiaron, y fueron remitidas (al ISS casi todas) para un tratamiento definitivo.

\section{Análisis de valores relativos}

Los valores para la sensibilidad, especificidad, valor predictivo positivo y valor predictivo negativo para cáncer de las pruebas evaluadas utilizando el resultado de la anatomía patológica o el conocimiento del tratamiento definitivo para cáncer como prueba validatoria, fueron los siguientes:

Tabla 9

\begin{tabular}{|lccccc|}
\hline Prueba & $\mathbf{n}$ & Sensibilidad & Especificidad & V.P. positivo & V.P. negativo \\
\hline ACAF & 246 & $87.8(80.1-92.9)$ & $98.5(94.0-99.7)$ & $98.1(92.5-99.7)$ & $90.2(83.8-94.3)$ \\
DX. Clínico & 313 & $92.7(86.2-96.4)$ & $83.7(77.5-88.5)$ & $78.6(70.9-84-8)$ & $94.6(89-8-97.4)$ \\
Mamografía & 214 & $82.7(73.4-89.3)$ & $77.6(68.7-84.6)$ & $75.7(66.3-83-2)$ & $84.1(75.5-90.2)$ \\
\hline
\end{tabular}

El diagnóstico clínico presenta los valores más altos en sensibilidad y valor predictivo negativo, siendo más alto el de éste último. Esto podría interpretarse como las masas palpables de mama de carácter maligno presentando características clínicas que permiten identificarlas como tales en una alta proporción de casos. Hay que destacar el alto valor de especificidad y valor predictivo positivo de la ACAF, prueba que se presenta entonces como la de menor probabilidad de errar en el diagnóstico de malignidad frente a un resultado positivo. La mamografía, en este grupo de pacientes, presentó los más bajos valores para los cuatro parámetros evaluados cuando se consideran individualmente.

El análisis de las pruebas combinando el diagnóstico clínico con la ACAF o con la mamografía se presenta en la Tabla 10.

La combinación de ambas herramientas diagnósticas muestra una mejoría importante en todos los parámetros considerados, con resultados mejores cuando se combinan la presunción diagnóstica inicial y la ACAF, donde todos los parámetros muestran valores superiores al $97 \%$.
El clínico que, teniendo una sospecha de cáncer se encuentra frente a un resultado de ACAF positivo (valor predictivo positivo de la prueba), tiene una alta probabilidad de no equivocar su diagnóstico. Igualmente frente a la sospecha clínica del cáncer el uso de la ACAF se presenta como la mejor combinación para validar esa sospecha por su alta sensibilidad y especificidad. El hallazgo de altos valores predictivos negativos para las dos combinaciones las hace confiables para reafirmar la sospecha de una lesión benigna, siendo mejor la combinación de ACAF y sospecha diagnóstica de lesión benigna.

$\mathrm{Al}$ analizar los mismos parámetros pero considerando la positividad en la combinación de las pruebas como cualquiera de las dos positiva, los parámetros evaluados muestran valores menores en la especificidad y en el valor predictivo positivo, con descensos de alrededor del $15 \%$, como puede apreciarse en la Tabla 11.

La evaluación de las tres pruebas simultáneamente positivas para cáncer muestran valores cercanos al $100 \%$ en todos los parámetros. Tabla 12.

Tabla 10

\begin{tabular}{|lccccc|}
\hline DX CL+ & n & Sensibilidad & Especificidad & V.P. positivo & V.P. negativo \\
\hline ACAF & 246 & $87.8(80.1-92.9)$ & $98.5(94.0-99.7)$ & $98.1(92.5-99.7)$ & $90.2(83.8-94.3)$ \\
Mamografía & 214 & $82.7(73.4-89.3)$ & $77.6(68.7-84.6)$ & $75.7(66.3-83-2)$ & $84.1(75.5-90.2)$ \\
\hline
\end{tabular}


Tabla 11

\begin{tabular}{|lccccc|}
\hline DX CL+ & $\mathbf{n}$ & Sensibilidad & Especificidad & V.P. positivo & V.P. negativo \\
\hline ACAF & 235 & $98.2(92.9-99.7)$ & $85.6(77.9-91.0)$ & $85.7(78.1-91.1)$ & $98.2(92.9-99.7)$ \\
Mamografía & 203 & $95.7(88.8-98.6)$ & $67.0(57.2-75.5)$ & $71.4(62.6-78-9)$ & $94.8(86.5-98.3)$ \\
\hline
\end{tabular}

Tabla 12

\begin{tabular}{|lccccc|}
\hline Prueba & $\mathbf{n}$ & Sensibilidad & Especificidad & V.P. positivo & V.P. negativo \\
\hline Triple & 113 & $98.4(90.3-99.9)$ & $98.0(88.0-99.9)$ & $98.4(90.3-99.9)$ & $98.0(88.0-99.9)$ \\
\hline
\end{tabular}

\section{Lesiones palpables no biopsiadas - Seguimiento}

De las 689 lesiones benignas no biopsiadas, se efectuaron 226 controles clínicos en 218 pacientes (32.8\%), en un corte hecho en diciembre 20 de 1995.

Un total de $104 \mathrm{ACAF}$ se practicaron a estas pacientes al año o más de la primera punción (10 resultados aparecen pendientes) y se ordenaron 62 mamografías de las cuales el $28.0 \%$ están pendientes.

\section{Cambios fibroquísticos}

334 lesiones diagnosticadas clínicamente como CFQ no se biopsiaron. De ellas 126 se presentaron como un nódulo dominante y 158 como una placa densa y en 51 no se consignó este dato. El diagnóstico de la ACAF fue coincidente en el 44\% de las lesiones: Enfermedad proliferativa benigna $33.3 \%$ y enfermedad no proliferativa $11.0 \%$. Ocho lesiones con atipias leves no se biopsiaron y de ellas se les hizo seguimiento a cinco sin encontrar ningún cambio.

De los 334 CFQ con ACAF, 112 lesiones se pudieron seguir $(33.3 \%)$ en un lapso de 4 a 33 meses, con un promedio de 17.6 meses. 43 lesiones se mostraron estables, 42 desaparecieron, 15 disminuyeron de tamaño y 12 aumentaron.

Se les practicó 39 ACAF de control de las cuales el $28.2 \%$ reportaron muestra insuficiente, y el $20.3 \%$ de los casos "mama". Es muy posible que una vez descartadas las pacientes biopsiadas, los diagnósticos de cambio fibroquístico restantes obedecieron a hallazgos menores. Esto pudiera explicar en parte los resultados insuficientes. De igual manera la fibrosis estromal, los lipomas o fibroadenolipomas, las cicatrices, son todas lesiones que dieron ACAF insuficientes o compatibles con tejido mamario escaso pero normal -"mama"-, pero pueden en un momento dado ser de gran utilidad para aumentar la certeza diagnóstica en los casos de patología benigna, no sólo por la lectura del contenido (grasa, fibroblastos, histiocitos), sino por la sensación que se tiene al adelantar y devolver la aguja dentro de la lesión experiencia descrita por algunos autores (16).

\section{Fibroadenomas}

220 lesiones con diagnóstico clínico de FA quedaron en observación. Las pacientes estuvieron de acuerdo con esta opción y se comprometieron para asistir al control. Algunas pacientes con FA de tamaño importante, rehusaron la resección del nódulo, y tres motivadas para el seguimiento se operaron en otras instituciones y el resultado fue "benigno". El $43.1 \%$ de las ACAF de esas pacientes eran compatibles con FA, el $10.0 \%$ con EPB, el $10.0 \%$ con benigno, el $12.2 \%$ de las muestras fueron insuficientes y el $15.0 \%$ reportaron "mama" o mama normal. De los $220 \mathrm{FA}, 83$ se siguieron $(37.7 \%)$, en un lapso de 3 a 32 meses, promedio de $17.1 \%$. 31 lesiones permanecieron estables (37.3\%), 28 aumentaron (33.7\%), seis disminuyeron (7.2\%) y 18 desaparecieron (21.6\%), hallazgos estos que reflejan la historia natural de los FA (17). Fueron dadas de alta 15 pacientes, seis resecciones se indicaron: tres por aumento de tamaño y tres por presencia de hiperplasia epitelial en la ACAF de los cuales se biopsiaron tres, con resultados de histopatología de FA.

A este grupo se les realizó 49 ACAF de control, obteniéndose un $44.8 \%$ de compatibilidad con el diagnóstico de FA, $16.3 \%$ de enfermedad proliferativa benigna con tres hiperplasias e igual porcentaje para "mama" e insuficiente. 26 lesiones fueron evaluadas con mamografía y todos los resultados fueron negativos para CA.

En vista de la dificultad para reclutar las pacientes para el seguimiento clínico, los investigadores iniciaron la aplicación de la encuesta telefónica descrita a partir de noviembre de 1995, la cual no fue evaluada aún.

\section{Conclusiones}

Evaluados el diagnóstico clínico, la aspiración citología con aguja fina y la mamografía en el diagnóstico de las lesiones palpables de la mama en la consulta de patología mamaria de la institución X, se concluye que la impresión clínica acompañada de una aspiración biopsia citología ACAF, resultó ser la mejor manera de diagnosticar la naturaleza de una lesión, para decidir una conducta terapéutica adecuada, o para optar por un seguimiento clínico, y esto se logró en la primera visita al médico, a muy bajo costo, sin morbilidad, sin demora y con una excelente aceptación por parte de las pacientes.

Aunque el entrenamiento es importante tanto para el que practica la punción aspiración, como para el citopatólogo, la técnica no ofreció ninguna dificultad importante, y la sensibilidad, especificidad, VPP y VPN 
de la prueba coincidieron con lo reportado en la literatura para los centros de referencia, excepto por el porcentaje de muestras insuficientes que en este trabajo fue alto (35.6\%).

El seguimiento clínico de 226 lesiones no demostró ningún caso de carcinoma no diagnosticado. Esta cifra constituye el $30 \%$ de las lesiones no biopsiadas.

Para una población institucional afectada de cáncer, con lesiones en su mayoría mayores de 4 centímetros en el momento de la primera consulta, la ACAF parece ser suficiente para establecer el tratamiento definitivo puesto que casi siempre coincidió con un diagnóstico clínico y mamográfico positivo para cáncer. El ahorro de más de 40 biopsias en estas pacientes es significativo, si se tiene en cuenta que una ACAF y una consulta médica en la institución X tienen un costo actual de US 10 cada una.

En las actuales condiciones del ejercicio médico, altamente competitivo en calidad y costo-efectividad, la evaluación de la ACAF demostró que fue en interacción con el diagnóstico clínico más eficaz en todos los aspectos que una biopsia convencional, para el diagnóstico de CA y patología benigna y que practicada a todas las lesiones palpables permitió mejorar la relación malignidad-benignidad de los especímenes de biopsias en la institución X, al 30.6\%, evitó en gran parte la morbilidad inherente a la biopsia al evitar procedimientos inadecuados, por permitir planearlos mejor y abarató los costos de la atención a las pacientes.

En cuanto a la mamografía aplicada en la llamada triple prueba, aumentó el porcentaje de las cuatro variables examinadas en solamente $0.2 \%$ a $0.5 \%$. Dado que los costos de la mamografía son mayores que la ACAF y que los hallazgos requieren confirmación histológica, la citología resulta más efectiva para el diagnóstico de un tumor palpable, y la mamografía para evaluar el cáncer ya diagnosticado en cuanto a su bilateralidad o multicentridad, lo que es necesario para indicar un tratamiento. En pacientes mayores de 35 años con patología benigna, la mamografía continúa siendo la prueba de tamizaje más importante para descartar un cáncer no palpable asociado, y se debe indicar.

El hecho de que el clínico confrontara resultados sospechosos de la mamografía con la clínica de los pacientes y les asignara una categoría benigna, muestra la importancia de la comunicación entre el radiólogo y el médico tratante.

\section{BIBLIOGRAFIA}

1. Wilkinson E et al. Technics and results of aspiration citology for diagnosis of benign and malignant disease of the breast. Surgical Clinics of North America. 1990; 70(4): 801-813.

2. Costa MJ et al. Breast fine needle aspiration cytology utility as a screening tool for clinically palpable lesions. Acta Cytologica. 1993; 37(4): 461-469.

3. Ciatto $S$ et al. Fine-needle cytology of the breast; e controlled study of aspiration versus non aspiration. Diagnostic-Cytopathology. 1991; 7(2): $125-127$.

4. Ciatto S. On behalf of detection and diagnosis study grope of the European Society of Mastology (Shortened version of original quidelines, Meeting of a Eusoma. Venice March 1991). The Brecest. 1992; 1: 207-210.

5. Glober SP et al. Preoperative evaluation of palpable breast tumors. Sur Afric-Journal Surgery. 1990; 28(4): 198-292.

6. Hermansen $\mathrm{C}$ et al. Diagnostic reliability of combined physical examination, mamography and fine needley puneture ("triple test") in breast tumors: A prospective study cancer 1987; 60-1866.

7. Haagensen C. Enfermedades de la mama. $3^{\text {a }}$ Ed. Panamericana.
8. Page D., Anderson. Diagnostic histopatology of the breast 1992. Copia de la $2^{a}$ Ed. 1998.; 120-156.

9. Rosen Paul Peter., Overman Harold. Fascículo 7. Armed Forcet Institute of Pathology, 1993; 49-65.

10. Mirjana $\mathrm{M}$ et al. Significance of proliferative epithelial changes in breast fine needle aspiration. Cancer. 1992; 70(4): 781-783.

11. Ciatto $S$ et al. Análisis de 22.063 aspiraciones citología con aguja fina en diez laboratorios italianos.

12. Haagensen C. Enfermedades de la mama $3^{\text {a }}$ ed. Panamericana. p. 823.

13. Lynn A., Rogers et al. Breast carcinoma, simulating fibroadenoma or fibrocystic change by fine-needle aspiration. American Journal, Clinical Pathology. 1992; 98: 155-160.

14. Wilkinson EJ et al. Cytological needle sampling of the breast. Techniques and results in the breast. (Ed. Bland and Copeland) Swinders Company. 1991; 475.

15. Donegan WL., Sratt JS. Cáncer de mama. Buenos Aires. Panamericana. 1982; 46.

16. Roberts JC., Rainsbury RM. Tactile sensation: a new clinical sign during fine needle aspiration of breast lumps. Annals Royal College. Surgery England. 1994; 76(2): 136-138. 